\title{
ON A CERTAIN FAMILY OF ASYMMETRIC RIEMANN SURFACES WITH THE CYCLIC AUTOMORPHISM GROUP
}

\author{
Ewa KozlowsKa-Walania AND Ewa TyszKowsKa
}

\begin{abstract}
A compact Riemann surface $X$ of genus $g \geq 2$ is called asymmetric or pseudo-real if it admits an anticonformal automorphism but no anticonformal involution. The order $d=\#(\delta)$ of an anticonformal automorphism $\delta$ of such a surface is divisible by 4 . In the particular case where $d=4, \delta$ is a pseudo-symmetry and the surface is called pseudo-symmetric.

A Riemann surface $X$ is said to be p-hyperelliptic if it admits a conformal involution $\rho$ for which the orbit space $X /\langle\rho\rangle$ has genus $p$. This notion is the particular case of so called cyclic $(q, n)$-gonal surface which is defined as the one admitting a conformal automorphism $\phi$ of prime order $n$ such that $X / \phi$ has genus $q$. We are interested in possible values of $n$ and $q$ for which an asymmetric surface of given genus $g \geq 2$ is $(q, n)$-gonal, and possible values of $p$ for which the surface is $p$-hyperelliptic. Up till now, this problem was solved in the case where the surface is asymmetric and pseudo-symmetric. If an asymmetric Riemann surface $X$ is not pseudo-symmetric then any anticonformal automorphism of $X$ has order divisible by $2^{s} n$ for $s \geq 3$ and $n=1$ or $n$ being an odd prime. In this paper we give the necessary and sufficient conditions on the existence of an asymmetric Riemann surface with the full automorphism group being $G=Z_{2 s}$, and we study $(q, n)$-gonal automorphisms and $p$-hyperelliptic involutions in $G$.
\end{abstract}

\section{Introduction}

A Riemann surface is called asymmetric, if it admits an anticonformal automorphism but no anticonformal involution. The term pseudo-real is also used, see for example [5]. Asymmetric Riemann surfaces appear naturally when we consider an involution $\imath: \mathscr{M}_{g} \rightarrow \mathscr{M}_{g}$ on the moduli space of Riemann surfaces of genus $g$ mapping a Riemann surface onto its complex conjugate.

2010 Mathematics Subject Classification. Primary 30F99; Secondary 14 H37.

Key words and phrases. Riemann surface, symmetry of Riemann surface, asymmetric Riemann surface, pseudo-symmetric Riemann surface, Fuchsian groups, NEC groups.

First author supported by the Research Grant NCN UMO-2015/17/B/ST1/03235 of the Polish National Science Centre.

Received September 15, 2015; revised January 19, 2016. 
Then $\imath$ preserves symmetric surfaces while asymmetric surfaces are outside the set $\operatorname{Fix}(\imath)$.

In the literature there are many results concerning asymmetric surfaces. We would like to mention some of them which were a starting point for this paper. In [8] asymmetric surfaces with cyclic automorphism groups are studied, and in [5] authors prove that there exists an asymmetric Riemann surface of any genus $g \geq 2$, they give the sharp bound on the order of its automorphism group, and they study asymmetric surfaces of genera 2 and 3 . Also, the minimal genus problem for cyclic actions $Z_{n}$ for arbitrary $n$ on pseudo-real surfaces was completely solved in [1] by Gromadzki and Baginski.

An orientation reversing automorphism which has order 4 is called a pseudosymmetry and the corresponding surface-pseudo-symmetric. Not every asymmetric surface is pseudo-symmetric - it is enough to consider an asymmetric surface with the full automorphism group being $Z_{8}$. Also, not every pseudosymmetric surface is asymmetric.

In this paper we study the degree of hyperellipticity of asymmetric Riemann surfaces with the full automorphism group being a cyclic group of order $2^{s} n$ for a prime integer $n$ and $s \geq 3$. A compact Riemann surface $X$ of genus $g \geq 2$ is called p-hyperelliptic if it admits a conformal involution (called a p-hyperelliptic involution) such that the orbit space under the action of this involution is an orbifold of genus $p$. In particular cases, when $p=0$ and $p=1$, the surface is called hyperelliptic and elliptic-hyperelliptic respectively. It was proved in [10], that for $g>4 p+1$, the $p$-hyperelliptic involution is unique and hence central in the group of all automorphisms of the surface. Using this result, groups of automorphisms of hyperelliptic, elliptic-hyperelliptic and 2-hyperelliptic surfaces were determined respectively in [6], [16] and [17]. In the asymmetric case, the hyperelliptic surfaces were studied in [15], whilst in [3] the authors determined the defining equations for such surfaces; the special case of hyperelliptic asymmetric pseudo-symmetric surfaces is also treated there. A $p$-hyperelliptic surface is the particular case of so-called cyclic $(q, n)$-gonal surface, which is defined as the one admitting a conformal automorphism $\delta$ of prime order $n$ such that $X / \delta$ has genus $q$.

This paper is a continuation of [11], where we studied asymmetric Riemann surfaces with the full automorphism group $Z_{4 n}$. We gave the necessary and sufficient conditions on the existence of such a surface, we determined the degree of its hyperellipticity, and we found all integers $q$ for which the surface is $(q, n)$ gonal. Let us notice that an asymmetric Riemann surface whose full automorphism group is a cyclic group $Z_{2^{s} n}$ is pseudo-symmetric only for $s=2$. So in this paper we consider surfaces which are not pseudo-symmetric.

\section{Preliminaries}

In this chapter we give the basic definitions concerning theory of noneuclidean crystallographic groups, called NEC groups, which are applied in the paper. The NEC groups are discrete and cocompact subgroups of the group $\mathscr{G}$ 
of all isometries of the hyperbolic plane $\mathscr{H}$. For any NEC group $\Lambda$, its algebraic structure is described by the so-called signature:

$$
s(\Lambda)=\left(h ; \pm ;\left[m_{1}, \ldots, m_{r}\right] ;\left\{\left(n_{11}, \ldots, n_{1 s_{1}}\right), \ldots,\left(n_{k 1}, \ldots, n_{k s_{k}}\right)\right\}\right),
$$

where the brackets $\left(n_{i 1}, \ldots, n_{i s_{i}}\right)$ are called the period cycles and the integers $n_{i j}$ are the link periods, $m_{i}$ are called the proper periods and $h$ is the orbit genus of $\Lambda$.

A presentation for the group $\Lambda$ with signature (1) is given by the following generators, called canonical generators:

$x_{1}, \ldots, x_{r}, e_{i}, c_{i j}$, for $1 \leq i \leq k, 0 \leq j \leq s_{i}$, and $a_{1}, b_{1}, \ldots, a_{h}, b_{h}$ if the sign is + or $d_{1}, \ldots, d_{h}$ otherwise,

and relations:

$$
\begin{gathered}
x_{i}^{m_{i}} \text { for } 1 \leq i \leq r, c_{i j-1}^{2}, c_{i j}^{2},\left(c_{i j-1} c_{i j}\right)^{n_{i j}}, c_{i 0} e_{i}^{-1} c_{i s_{i}} e_{i} \text {, for } 1 \leq i \leq k, 1 \leq j \leq s_{i} \text { and } \\
x_{1} \cdots x_{r} e_{1} \cdots e_{k} a_{1} b_{1} a_{1}^{-1} b_{1}^{-1} \cdots a_{h} b_{h} a_{h}^{-1} b_{h}^{-1} \quad \text { or } \quad x_{1} \cdots x_{r} e_{1} \cdots e_{k} d_{1}^{2} \cdots d_{h}^{2},
\end{gathered}
$$

according to whether the sign is + or -. The last relation is called the long relation. The elements $x_{i}$ are elliptic transformations, $a_{i}, b_{i}$ hyperbolic translations, $d_{i}$ glide reflections and $c_{i j}$ hyperbolic reflections.

An abstract group with the presentation given above can be realized as an NEC group $\Lambda$ if and only if the value

$$
\eta h+k-2+\sum_{i=1}^{r}\left(1-\frac{1}{m_{i}}\right)+\frac{1}{2} \sum_{i=1}^{k} \sum_{j=1}^{s_{i}}\left(1-\frac{1}{n_{i j}}\right),
$$

is positive, where $\eta=2$ or 1 according to the sign being + or - . The above expression times $2 \pi$ is equal to the hyperbolic area $\mu(\Lambda)$ of a fundamental region of $\Lambda$. The Riemann-Hurwitz formula says that for any subgroup $\tilde{\Lambda}$ of an NEC group $\Lambda$ with finite index,

$$
[\Lambda: \tilde{\Lambda}]=\frac{\mu(\tilde{\Lambda})}{\mu(\Lambda)} .
$$

If an NEC group has no orientation reversing elements, then it is the classical Fuchsian group and has a signature of the form

$$
\left(h ;+;\left[m_{1}, \ldots, m_{r}\right] ;\{-\}\right),
$$

which is often written as $\left(h ; m_{1}, \ldots, m_{r}\right)$.

Any NEC group $\Lambda$ with the signature (1) has so-called canonical Fuchsian subgroup $\Lambda^{+}$consisting of all orientation preserving elements which by [14], has signature

$$
\left(\eta h+k-1 ; m_{1}, m_{1}, \ldots, m_{r}, m_{r}, n_{11}, \ldots, n_{k s_{k}}\right) .
$$

A signature $\sigma$ is called maximal, if for every NEC group $\Lambda^{\prime}$ with a signature $\sigma^{\prime}$ containing an NEC group $\Lambda$ with the signature $\sigma$ and having the same 
Teichmüller dimension, the equality $\Lambda=\Lambda^{\prime}$ holds. If the above condition does not hold, then the pair $\left(\sigma, \sigma^{\prime}\right)$ is called a normal or non-normal pair according to if $\Lambda$ is a normal subgroup of $\Lambda^{\prime}$ or not. The complete lists of normal and non-normal pairs are given in [2] and [9] (see also [13] and [4]). An NEC group $\Lambda$ is called maximal if there does not exist another NEC group containing it properly. Also, for any maximal signature $\sigma$, there exists a maximal NEC group with the signature $\sigma$.

A torsion-free Fuchsian group $\Gamma$ is called the surface Fuchsian group. It has a signature $(g ;-)$, and $\mathscr{H} / \Gamma$ is a compact Riemann surface of genus $g$. Conversely, every compact Riemann surface $X$ of genus $g \geq 2$ is isomorphic to such an orbit space for some surface Fuchsian group $\Gamma$. A finite group $G$ is a group of automorphisms of $X$ if and only if $G=\Lambda / \Gamma$ for some NEC group $\Lambda$ containing $\Gamma$ as a normal subgroup, and we say that $G$ acts with the signature $s(\Lambda)$. If $G$ is not the full automorphism group of $X$, then there exists another NEC group $\Lambda^{\prime}$ normalizing $\Gamma$ such that $\Lambda \subsetneq \Lambda^{\prime}$ with a finite index.

The formula on the number of fixed points of an automorphism of a Riemann surface was given by Macbeath in [12]. By $N_{G}(\langle g\rangle)$ we mean the normalizer in $G$ of the subgroup generated by $g$.

THeOREM 2.1. Let $G=\Delta / \Gamma$ be the group of orientation preserving automorphisms of a Riemann surface $X=\mathscr{H} / \Gamma$, and let $x_{1}, x_{2}, \ldots, x_{r}$ be the set of canonical elliptic generators of $\Delta$ with periods $m_{1}, \ldots, m_{r}$ respectively. Let $\theta: \Delta \rightarrow G$ be the canonical epimorphism. Then the number $m$ of points of $X$ fixed by $g \in G$ is given by the formula

$$
m=\left|N_{G}(\langle g\rangle)\right| \sum 1 / m_{i}
$$

where the sum is taken over those $i$ for which $g$ is conjugate to a power of $\theta\left(x_{i}\right)$.

\section{The degree of hyperellipticity}

Let $X=\mathscr{H} / \Gamma$ be an asymmetric Riemann surface of genus $g \geq 2$, for a surface Fuchsian group $\Gamma$. Since $X$ has anticonformal automorphisms but no symmetries, it follows that the group of automorphisms of $X$ acts with a signature of the form $\left(h ;-;\left[m_{1}, \ldots, m_{r}\right] ;\{-\}\right)$. Moreover, the order $\alpha$ of an anticonformal automorphism $\varphi$ of $X$ is divisible by 4 , since if $\alpha$ is odd, then $\varphi$ is a power of $\varphi^{2}$ and so it would be orientation preserving, while if $\alpha \equiv 2$ (4) then $\varphi^{\alpha / 2}$ would be a symmetry of $X$.

For a prime divisor $n$ of $\alpha$, the element $\rho=\varphi^{\alpha / n}$ is a $(q, n)$-gonal automorphism of $X$, where $q$ is the genus of the orbifold $X /\langle\rho\rangle$. The group $\langle\rho\rangle$ acts with the signature $(q ; n . r ., n)$, where by the Riemann-Hurwitz formula $r=2+(2 g-2 n q) /(n-1)$. Thus according to Theorem 2.1, $\rho$ has $r$ fixed points.

We want to determine the possible values of $q$ in the case when $\rho$ is an involution. The task becomes much easier, if we consider a subgroup of $\langle\varphi\rangle$ 
generated by an anticonformal automorphism $\delta=\varphi^{\alpha /\left(2^{s} n\right)}$, where $s$ is the greatest integer such that $2^{s}$ divides $\alpha$, and $n$ is an odd prime divisor of $\alpha$ or $n=1$ if $\alpha=2^{s}$. The possible degrees of hyperellipticity in the case $s=2$ were given in [11], where we also proved that no cyclic group acts on an asymmetric Riemann surface with a non-maximal signature. Here we shall study surfaces which are not pseudo-symmetric and therefore we shall assume that $s \geq 3$. The notation introduced above will be consistently employed throughout the chapter, unless directly stated otherwise. First, we give the necessary and sufficient conditions on the existence of an asymmetric Riemann surface of genus $g$ with the automorphism group $Z_{2^{s} n}$.

THEOREM 3.1. A cyclic group $Z_{2^{s_{n}}}$ is the full automorphism group of an asymmetric Riemann surface of genus $g$ if and only if $g=n q+a(n-1)$ for some integers $a \geq-1, q \geq 0$, and there exist nonnegative integers $k, \gamma, k_{i}, l_{i}$, $i=1, \ldots, s-1$ such that

$$
\begin{gathered}
a=2^{s-1} k+\sum_{i=1}^{s-1} 2^{s-1-i} k_{i}-1 \quad \text { for } n \neq 1 \\
q=1+2^{s-1}\left[\gamma-1+\sum_{i=1}^{s-1}\left(l_{i}+k_{i}\right)\right]-\sum_{i=1}^{s-1} 2^{s-1-i}\left(l_{i}+k_{i}\right),
\end{gathered}
$$

(III) $k=k_{1}=\cdots=k_{s-1}=0$ for $n=1$,

(IV) $\gamma$ has the same parity as $g$,

$(\mathrm{V})$ if $\gamma=0$, then $l_{s-1} \neq 0$ for $n=1 ; k_{s-1} \neq 0$ or $k l_{s-1} \neq 0$ for $n \neq 1$, and none of the two cases holds:

(a) $\gamma=1, l_{s-1}=k_{s-1}=0$ and $\sum_{i=1}^{s-2}\left(l_{i}+k_{i}\right)+k=1$,

(b) $\gamma=0, l_{s-1}+k_{s-1}=1$ and $\sum_{i=1}^{s-2}\left(l_{i}+k_{i}\right)+k=1$.

Proof. Suppose that $\delta$ is an anticonformal automorphism of order $2^{s} n$ of an asymmetric Riemann surface $X=\mathscr{H} / \Gamma$ of genus $g$, and let $G=\langle\delta\rangle$. Then there exists an NEC group $\Lambda$ containing $\Gamma$ as a normal subgroup with index $2^{s} n$ such that $G=\Lambda / \Gamma$. Since there is no period cycles in the signature of $\Lambda$ and the conformal automorphisms in $G$ have orders $2^{i}, n$ or $2^{i} n$ for some $i=1, \ldots, s-1$, it follows that $\Lambda$ has a signature of the form

$$
\left(\gamma+1 ;-;\left[(2)^{l_{1}},(4)^{l_{2}}, \ldots,\left(2^{s-1}\right)^{l_{s-1}},(n)^{k},(2 n)^{k_{1}}, \ldots,\left(2^{s-1} n\right)^{k_{s-1}}\right] ;\{-\}\right),
$$

where $k, \gamma, l_{i}$ and $k_{i}$ are nonnegative integers, and the symbol $(m)^{r}$ denotes $r$ periods equal to $m$. In the particular case when $n=1, k=k_{1}=\cdots=k_{s-1}=0$. By the Riemann-Hurwitz formula,

$$
g=1+2^{s-1}\left[n \gamma-n+n \sum_{i=1}^{s-1}\left(l_{i}+k_{i}\right)+k(n-1)\right]-\sum_{i=1}^{s-1} 2^{s-1-i}\left(n l_{i}+k_{i}\right) .
$$


According to (2), the canonical Fuchsian subgroup $\Lambda^{+}$of $\Lambda$ has the signature

$$
\left(\gamma ;(2)^{2 l_{1}},(4)^{2 l_{2}}, \ldots,\left(2^{s-1}\right)^{2 l_{s-1}},(n)^{2 k},(2 n)^{2 k_{1}}, \ldots,\left(2^{s-1} n\right)^{2 k_{s-1}}\right) .
$$

The group $G^{+}=\Lambda^{+} / \Gamma=Z_{2^{s-1} n}$ is generated by the conformal automorphism $\delta^{2}$. If $n \neq 1$, then $\rho=\delta^{2^{s}} \in G^{+}$is a $(q, n)$-gonal automorphism of $X$ with $m=$ $2+(2 g-2 n q) /(n-1)$ fixed points, where $q$ is the genus of $X /\langle\rho\rangle$. On the other hand, by Macbeath's theorem, $m=2^{s} k+\sum_{i=1}^{s-1} 2^{s-i} k_{i}$ and so

$$
\begin{aligned}
(n-1) 2^{s-1} k & =(n-1)\left(m / 2-\sum_{i=1}^{s-1} 2^{s-1-i} k_{i}\right) \\
& =n-1+g-n q-(n-1) \sum_{i=1}^{s-1} 2^{s-1-i} k_{i} .
\end{aligned}
$$

By substituting the last equation to (4), we get (II). Thus $g-n q=$ $(n-1)\left(2^{s-1} k+\sum_{i=1}^{s-1} 2^{s-1-i} k_{i}-1\right)=a(n-1)$ for $a$ given by (I).

By inspecting lists in [2] and [9], we check that the signature (3) is nonmaximal if and only if it has a form $(2 ;-;[t] ;\{-\})$ or $(1 ;-;[t, u] ;\{-\})$ for some $u \in\left\{2^{s-1}, 2^{s-1} n\right\}$ and $t \in\left\{n, 2^{i} n, 2^{i}\right\}$, where $i \neq s-1$. However, it was proved in [4] that no cyclic group acts on an asymmetric Riemann surface with listed signatures, as they in fact give rise to actions on symmetric surfaces. Thus the cases (a) and (b) must be excluded.

The action of $G$ on $X$ is induced by an epimorphism $\theta: \Lambda \rightarrow G$ with torsionfree kernel $\Gamma$. Any such an epimorphism maps elliptic generators $x_{i} \in \Lambda$ of orders $m_{i}$ to $\delta^{2^{s} n \gamma_{i} / m_{i}}$ for some integers $\gamma_{i}$ co-prime with $m_{i}$. Thus the product of all elliptic generators is mapped to $\delta^{T}$ for some even integer $T$. The glide reflection generators $d_{i}$ of $\Lambda$ are mapped to $\delta^{t_{i}}$ for some odd integers $t_{i}$. Thus the long relation is preserved if and only if the sum of $\gamma+1$ odd integers $t_{i}$ is equal to $-T / 2$ modulo $2^{s} n$. The parity of the last integer depends only on the parity of $l_{s-1}+k_{s-1}$ and so by (4), is different from the parity of $g$. Thus $\gamma$ must have the same parity as $g$. Let us also notice, that for $\gamma=0$, the restriction of $\theta$ to $\Lambda^{+}$is an epimorphism onto $Z_{2^{s-1} n}$ under condition that $l_{s-1} \neq 0$ for $n=1$, and $k_{s-1} \neq 0$ or both $k$ and $l_{s-1}$ are different from 0 for $n \neq 1$.

Conversely, suppose that $g=n q+a(n-1)$ for some integers $a \geq-1$ and $q \geq 0$, and there exist nonnegative parameters $l_{1}, \ldots, l_{s-1}, k_{1}, \ldots, k_{s-1}, k$ and $\gamma$ such that the conditions (I) $-(\mathrm{V})$ are satisfied, and neither case (a) nor (b) holds. Then an NEC group $\Lambda$ with the signature (3) is maximal. Let $\theta: \Lambda \rightarrow Z_{2^{s} n}=$ $\langle\delta\rangle$ be a homomorphism which maps the elliptic generators $x_{i}$ of orders $m_{i}$ to $\delta^{2^{s} n / m_{i}}$ and the glide reflection generators $d_{i}$ to $\delta^{t_{j}}$, for $\left(t_{1}, t_{2}, \ldots, t_{\gamma-2}, t_{\gamma-1}, t_{\gamma}, t_{\gamma+1}\right)$ $=(1,-1, \ldots,-1,1,-1,-T / 2)$ or $(1,-1, \ldots, 1,-1,-1,-T / 2+1)$, according to if $g$ is even or odd. Then $\theta$ is an epimorphism with torsion-free kernel $\Gamma$. By the Riemann-Hurwitz formula, the orbit space $X=\mathscr{H} / \Gamma$ has genus $g$, and $G=\Lambda / \Gamma$ is the full automorphism group of $X$. Since $G$ contains anticonformal automorphisms but no symmetries, it follows that $X$ is asymmetric. 
In the next theorem we exceptionally let the parameter $s$ have value 2 .

THEOREM 3.2. Suppose that $G=Z_{2^{s_{n}}}=\langle\delta\rangle$ is the automorphism group of an asymmetric Riemann surface $X$ of genus $g$, for $g \neq n-1,2 n-1,2 n-2$, and let $r=g \bmod n$. If $n>1$ and $g \geq(n-r)(n-1)$, then $X$ is a n-sheeted covering of an orbifold of genus $q$ ramified over $m$ points, where

$$
q=[g-(n-r)(n-1)] / n-c(n-1) \text { and } m=2[n(c+1)+1-r],
$$

for some integer $c \geq-1$ for which $q$ and $m$ are nonnegative. Moreover, for any such $c$, there exists an asymmetric Riemann surface of genus $g$ with the full automorphism group $Z_{4 n}$.

Proof. Suppose that $X /\left\langle\delta^{2^{s}}\right\rangle$ has genus $q$. Then $\delta^{2^{s}}$ is a $(q, n)$-gonal automorphism with $m=2+(2 g-2 n q) /(n-1)$ fixed points. By the proof of Theorem 3.1, $m$ is even and so $g=n q+a(n-1)$ for $a=m / 2-1$. Writing $g$ in the form

$$
g=[(g-r) / n+1-(n-r)] n+(n-r)(n-1),
$$

we can take $q=[g-(n-r)(n-1)] / n$ and $a=n-r$. However, there are other possibilities: $q=[g-(n-r)(n-1)] / n-c(n-1)$ and $a=n-r+c n$ for any integer $c \geq-1$ for which $q \geq 0$ and $a \geq-1$.

Now for a given such $c$, let $\gamma=q \bmod 2$, and let $k=0$ or -1 according to if $a$ is even or odd. Then there exists an NEC group $\Lambda$ with the signature

$$
\left(\gamma+1 ;-;\left[2,{ }^{q-2 \gamma-k}, 2, n,{ }^{(a-k)} \cdot{ }^{2}, n, 2 n, k+1,2 n\right] ;\{-\}\right)
$$

which is maximal for $(\gamma, q, a, k) \neq(1,1,1,-1),(0,0,1,-1)$ and $(0,0,2,0)$. In the exceptional cases, $g=2 n-1, n-1$ and $2 n-2$ respectively, however these values of $g$ are rejected by assumption. Let $\theta: \Lambda \rightarrow G=Z_{4 n}=\langle\delta\rangle$ be an epimorphism defined by

$$
\begin{array}{ll}
\theta\left(x_{i}\right)=\delta^{2 n}, & i=1, \ldots, k_{1}=q-2 \gamma-k, \\
\theta\left(x_{k_{1}+i}\right)=\delta^{4}, & i=1, \ldots, k_{2}=(a-k) / 2, \\
\theta\left(x_{k_{1}+k_{2}+i}\right)=\delta^{2}, & i=1, \ldots, k_{3}=k+1,
\end{array}
$$

and $\theta\left(d_{1}\right)=\delta^{-l}$ if $q$ is even, or $\theta\left(d_{1}\right)=\delta$ and $\theta\left(d_{2}\right)=\delta^{-1-l}$ if $q$ is odd, where $l=n q-n k+a+1-2 n \gamma$. Then the kernel $\Gamma$ of $\theta$ is a surface Fuchsian group with the signature $(g ;-)$ and $X=\mathscr{H} / \Gamma$ is an asymmetric Riemann surface $X$ of genus $g$ with the full automorphism group $Z_{4 n}=\Lambda / \Gamma$.

THEOREM 3.3. For any integer $g$ such that $g \geq 2^{s-1} n\left(2^{s-1}+n\right)$, there exists an asymmetric Riemann surface of genus $g$ with the full automorphism group $Z_{2} s_{n}$. 
Proof. Suppose that $g \geq 2^{s-1} n\left(2^{s-1}+n\right)$, and let $r=g \bmod n$,

$$
r^{\prime}=(g-r) / n+1 \bmod 2^{s-1} \text { and } b=\left[(g-r) / n+1-r^{\prime}\right] / 2^{s-1} .
$$

Then $g \geq 2^{s-1} n\left(2^{s-1}+n\right)-(n-r) \quad$ and $\quad$ so $\quad(g-r) / n+1 \geq 2^{s-1}\left(2^{s-1}+n\right) \geq$ $2^{s-1}\left(2^{s-1}+n-1\right)+r^{\prime}$. Thus

$$
b=\left[(g-r) / n+1-r^{\prime}\right] / 2^{s-1} \geq 2^{s-1}+n-1 \geq\left(2^{s-1}-r^{\prime}\right)+(n-r)-1 .
$$

For $b$ satisfying the last inequality, there exist parameters $a, q, \gamma, k, k_{i}, l_{i}$, $i=1, \ldots s-1$ for which the conditions $(\mathrm{I})-(\mathrm{V})$ of Theorem 3.1 are satisfied and neither case (a) nor (b) holds. Indeed, since

$$
\begin{aligned}
g & =[(g-r) / n+1-(n-r)] n+(n-r)(n-1) \\
& =\left[(b+1) 2^{s-1}-\left(2^{s-1}-r^{\prime}\right)-(n-r)\right] n+(n-r)(n-1),
\end{aligned}
$$

it follows that $g=n q+a(n-1)$ for $q=(b+1) 2^{s-1}-\left(2^{s-1}-r^{\prime}\right)-(n-r)$ and $a=n-r$. The remaining parameters can be chosen as follows:

for $b$ odd

$$
\begin{array}{ll}
n \neq 1: & \gamma=b+1-(n-r)-\left(2^{s-1}-r^{\prime}\right), k=0, k_{s-1}=n-r+1, \\
& l_{s-1}=2^{s-1}-r^{\prime} \text { and } k_{i}=l_{i}=0 \text { for } i \neq s-1, \\
n=1: & \gamma=b-\left(2^{s-1}-r^{\prime}\right), l_{s-1}=2^{s-1}-r^{\prime}+2, l_{i}=0 \text { for } i \neq s-1, \\
\text { for } b \text { even } & \\
n \neq 1: & \gamma=b+2-(n-r)-\left(2^{s-1}-r^{\prime}\right), k=0, k_{s-1}=n-r-1, \\
& k_{s-2}=1, l_{s-1}=2^{s-1}-r^{\prime}, l_{s-2}=0, \text { and } k_{i}=l_{i}=0 \\
& \text { for } i \neq s-1, s-2, \\
n=1: & \gamma=b+1-\left(2^{s-1}-r^{\prime}\right), l_{s-1}=2^{s-1}-r^{\prime}, l_{s-2}=1, \\
& l_{i}=0 \text { for } i \neq s-1, s-2 .
\end{array}
$$

Let us notice that by (6), $g$ has a different parity than the number $r+r^{\prime}$ and so $\gamma$ has the same parity as $g$. Thus by Theorem 3.1, there exists an asymmetric Riemann surface of genus $g$ with the full automorphism group $Z_{2} s_{n}$.

Remark 3.4. Suppose that $G=Z_{2^{s_{n}}}=\langle\delta\rangle$ acts on an asymmetric Riemann surface $X=\mathscr{H} / \Gamma$ of genus $g$ with the signature (3). Then $X$ is $p$-hyperelliptic for

$$
p=1+\left[n \gamma-n+n \sum_{i=1}^{s-1}\left(l_{i}+k_{i}\right)+k(n-1)\right] 2^{s-2}-\sum_{i=1}^{s-1} 2^{s-1-i}\left(n l_{i}+k_{i}\right),
$$

and if $n \neq 1$, then $X$ is $(q, n)$-gonal for $q$ given by (II).

Proof. If $\Lambda^{+} \leq \Lambda$ is the canonical Fuchsian subgroup, then $G^{+}=\Lambda^{+} / \Gamma=$ $Z_{2^{s-1} n}$ is the group of conformal automorphisms of $X$. By the proof of Theorem 3.1, $\delta^{2^{s}} \in G^{+}$is $(q, n)$-gonal automorphism of $X$ for $q$ given by (II). Moreover, $\rho=\delta^{2^{s-1} n} \in G^{+}$is $p$-hyperelliptic involution of $X$ with $m=2 g+2-4 p$ fixed points, where $p$ is the genus of $X /\langle\rho\rangle$. By Macbeath's theorem, $m=$ 
$\sum_{i=1}^{s-1} 2^{s-i}\left(n l_{i}+k_{i}\right)$. Thus

$$
p=\left[g+1-\sum_{i=1}^{s-1} 2^{s-1-i}\left(n l_{i}+k_{i}\right)\right] / 2,
$$

and so by (4), we get (7).

For any even nonnegative integer $u$, there exists an asymmetric Riemann surface $X$ with $(q, n)$-gonal automorphism and $p$-hyperelliptic involution having the same even number $u$ of fixed points.

THEOREM 3.5. Let $n \neq 1$. (i) Then for any even integer $u>0$, there exists an asymmetric Riemann surface of genus $g=1+\left(2^{s} n-1\right) \frac{u}{2}$ which is n-sheeted covering of an orbifold of genus $q=1+\left(2^{s}-1\right) \frac{u}{2}$ with $u$ ramification points and which is two-sheeted covering of an orbifold of genus $p=1+\left(2^{s-1} n-1\right) \frac{u}{2}$ also
with $u$ ramification points.

(ii) There exists an asymmetric Riemann surface of genus $g=2^{s} n+1$ which is unramified n-sheeted covering of an orbifold of genus $q=1+2^{s}$ and which is unramified two-sheeted covering of an orbifold of genus $p=1+2^{s-1} n$.

Proof. (i) For a given nonnegative even integer $u$, let $\Lambda$ be a maximal NEC group with the signature

$$
\left(u / 2+2 ;-;\left[2^{s-1} n, . \frac{u}{2} ., 2^{s-1} n\right] ;\{-\}\right),
$$

and let $G=\langle\delta\rangle$ be a cyclic group of order $2^{s} n$. Then there exists an epimorphism $\theta: \Lambda \rightarrow G$ given by $\theta\left(x_{i}\right)=\delta^{2}$ for $i=1, \ldots, \frac{u}{2}$, and $\theta\left(d_{j}\right)=\delta^{t_{j}}$, for $\left(t_{1}, \ldots, t_{u / 2+2}\right)=(1,-1, \ldots, 1,-1,-u / 2+1,-1)$ or $(1,-1, \ldots, 1,-1,-u / 2)$ according to if $u \equiv 0$ (4) or $u \equiv 2(4)$. The kernel $\Gamma$ of $\theta$ is a surface Fuchsian group which by the Riemann-Hurwitz formula has the signature $(g ;-)$, for $g=1+\left(2^{s} n-1\right) \frac{u}{2}$, and $G=\Lambda / \Gamma$ is the automorphism group of an asymmetric Riemann surface $X=\mathscr{H} / \Gamma$ of genus $g$. By Remark 3.4, $X$ has $p$-hyperelliptic involution and $(q, n)$-gonal automorphism for $q=1+\left(2^{s}-1\right) \frac{u}{2}$ and $p=$ $1+\left(2^{s-1} n-1\right) \frac{u}{2}$. The last elements have $2 g+2-4 p$ and $2+(2 g-2 n q) /(n-1)$ fixed points respectively, and these numbers are equal.

Similarly, we can prove the statement (ii) by taking an NEC group $\Lambda$ with the signature $(4 ;-;[-] ;\{-\})$ and an epimorphism $\theta: \Lambda \rightarrow G$ defined by $\theta\left(d_{1}\right)=$ $\theta\left(d_{2}\right)=\delta$ and $\theta\left(d_{3}\right)=\theta\left(d_{4}\right)=\delta^{-1}$.

An asymmetric Riemann surface of genus $g$ is $p$-hyperelliptic for some integer $p$ in the range $0 \leq p \leq \frac{g}{2}$ or $1 \leq p \leq \frac{g+1}{2}$, according to if $g$ is even of 
odd. We will prove that there are infinite sequences of $g$ for which the upper and lower bounds are attained.

THEOREM 3.6. For any $g$ such that $g \equiv 1\left(2^{s} n\right)$, there exists an asymmetric Riemann surface $X$ of genus $g$ with the full automorphism group $G=Z_{2^{s} n}$, which acts with one of signatures $\left(\frac{g-1}{2^{s-1} n}+2 ;-;[-] ;\{-\}\right)$ or $\left(2 ;-;\left[2, \frac{g-1}{2 \cdot s^{s-2 n}}, 2\right] ;\{-\}\right)$. In the first case, $X$ is $\frac{g+1}{2}$-hyperelliptic and in the second one, $X$ is elliptichyperelliptic.

Proof. If $g \equiv 1\left(2^{s} n\right)$, then $g=n q+a(n-1)$ for $q=(g-1) / n+1$ and $a=-1$. Let $k=l_{i}=k_{i}=0$ for $i=1, \ldots, s-1$ and $\gamma=(g-1) /\left(2^{s-1} n\right)+1$ or let $\gamma=1, k=l_{i}=k_{i}=0$ for $i=2, \ldots, s-1, l_{1}=(g-1) /\left(2^{s-2} n\right)$ and $k_{1}=0$. Then $\gamma, a, k, \gamma, k_{i}$ and $l_{i}$ satisfy the conditions $(\mathrm{I})-(\mathrm{V})$ of Theorem 3.1 and none of the cases (a), (b) holds. Thus there exists an asymmetric Riemann surface $X$ of genus $g$ with the full automorphism group $Z_{2^{s} n}$ acting with the signature (3). By Remark 3.4, $X$ is $p$-hyperelliptic, where $p=(g+1) / 2$ for the first choice of parameters, and $p=1$ for the second one.

THEOREM 3.7. For any $g$ such that $g \equiv 0\left(2^{s} n\right)$ there exists an asymmetric Riemann surface $X$ of genus $g$ with the full automorphism group $Z_{2^{s} n}$, which acts with one of the signatures $\left(\frac{g}{2^{s-1} n}+1 ;-;\left[2^{s-1} n\right] ;\{-\}\right)$ or $\left(1 ;-;\left[2, \stackrel{g}{2^{s-2 .} \cdot{ }^{2 .}}, 2,2^{s-1} n\right]\right.$; $\{-\})$. In the first case, $X$ is $\frac{g}{2}$-hyperelliptic and in the second one, $X$ is hyperelliptic.

Proof. Any integer $g$ satisfying the congruence $g \equiv 0\left(2^{s} n\right)$ can be written in the form $g=n q+a(n-1)$ for $q=g / n$ and $a=0$. If $n \neq 1$, then by Theorem 3.1, a cyclic group $Z_{2^{s_{n}}}$ acts on an asymmetric Riemann surface of genus $g$ with the signature (3), where $k=l_{i}=k_{i}=0$ for $i=1, \ldots, s-2, l_{s-1}=0, k_{s-1}=1$ and $\gamma=g /\left(2^{s-1} n\right)$, or $k=l_{i}=k_{i}=0$ for $i=2, \ldots, s-2, l_{1}=g /\left(2^{s-2} n\right), k_{1}=l_{s-1}=0$, $k_{s-1}=1$ and $\gamma=0$. If $n=1$, then we can assume that parameters $k, \gamma, k_{i}$ and $l_{i}, i=1, \ldots, s-2$ have above values, $k_{s-1}=0$ and $l_{s-1}=1$. By Remark 3.4, $X$ is $p$-hyperelliptic, where $p=g / 2$ in the first case and $p=0$ in the second one.

\section{REFERENCES}

[ 1 ] C. Baginski and G. Gromadzki, Minimal genus problem for pseudo-real Riemann surfaces, Arch. Math. 95 (2010), 481-492.

[2] E. Bujalance, Normal NEC signatures, Illinois J. Math. 26 (1982), 519-530.

[3] E. Bujalance and P. Turbek, Asymmetric and pseudo-symmetric hyperelliptic surfaces, Manuscripta Math. 108 (2002), 1-11.

[4] E. Bujalance AND M. D. E. Conder, On cyclic groups of automorphisms of Riemann surfaces, J. Lond. Math. Soc. 59 (1999), 573-584. 
[ 5 ] E. Bujalance, M. D. E. Conder and A. F. Costa, Pseudo-real Riemann surfaces and chiral regular maps, Trans. Amer. Math. Soc. 362 (2010), 3365-3376.

[6] E. Bujalance, J. M. Gamboa and G. Gromadzki, The full automorphisms group of hyperelliptic Riemann surfaces, Manuscripta Math. 79 (1993), 267-282.

[ 7 ] E. Bujalance, F. J. Cirre and G. Gromadzki, Groups of automorphisms of cyclic trigonal Riemann surfaces, J. Algebra 322 (2009), 1086-1103.

[ 8 ] J. J. Etayo Gordejuela, Nonorientable automorphisms of Riemann surfaces, Arch. Math. 45 (1985), 374-384.

[9] J. L. EstéveZ AND M. IzQuIERDo, Non-normal pairs of non-Euclidean grystallographic groups, Bull. Lond. Math. Soc. 38 (2006), 113-123.

[10] H. M. Farkas and I. Kra, Riemann surfaces, Grad. Texts Math., Springer-Verlag, 1980.

[11] E. Kozlowska-Walania and E. Tyszkowska, On asymmetric p-hyperelliptic Riemann surfaces, to appear in Ann. Acad. Sci. Fenn. Math.

[12] A. M. Macbeath, Action of automorphisms of a compact Riemann surface on the first homology group, Bull. Lond. Math. Soc. 5 (1973), 103-108.

[13] D. Singerman, Finitely maximal Fuchsian groups, J. Lond. Math. Soc. s2-6 (1972), $29-38$.

[14] D. Singerman, On the structure of non-euclidean crystallographic groups, Proc. Camb. Phil. Soc. 76 (1974), 233-240.

[15] D. Singerman, Symmetries and pseudo-symmetries of hyperelliptic surfaces, Glasg. Math. J. 21 (1980), 39-49.

[16] E. Tyszkowska, Topological classification of conformal actions on elliptic-hyperelliptic Riemann surfaces, J. Algebra 288 (2005), 345-363.

[17] E. Tyszkowska, Topological classification of conformal actions on 2-hyperelliptic Riemann surfaces, Bull. Inst. Math. Acad. Sin. 33 (2005), 345-368.

[18] E. Tyszkowska, Topological classification of conformal actions on cyclic p-gonal Riemann surfaces, J. Algebra 344 (2011), 296-312.

Ewa Kozłowska-Walania

INSTITUTE OF MATHEMATICS

FACUlTy OF Mathematics

Physics AND INFORMATICS

UNIVERSITY OF GDAŃSK

80-308 GDAŃSK

POLAND

E-mail: retrakt@mat.ug.edu.pl

Ewa Tyszkowska

INSTITUTE OF MATHEMATICS

FACUlTy OF MATHEMATICS

Physics AND INFORMATICS

UNIVERSITY OF GDAŃSK

80-308 GDAŃSK

POLAND

E-mail: ewa.tyszkowska@mat.ug.edu.pl 